\title{
Surgical management of a hand extravasation of anthracycline at late presentation
}

\author{
Komla Sena Amouzou ${ }^{*}$, Nabil Berny ${ }^{2}$, Tiemoko Moulaye Haidara², Abdessamad Chlihi², \\ Mohamed Ezzoubi \\ ${ }^{1}$ Department of Surgery, University of Lomé, Lomé, Togo \\ ${ }^{2}$ Department of Plastic Surgery, Hassan II University, Casablanca, Morocco
}

\begin{abstract}
Anthracycline extravasation remains a feared serious complication of chemotherapy. At late presentation, deep ulceration and extensive soft tissue damage are seen. Hand extravasation of anthracycline may lead to tendon and nerves destruction with functional and economical impairments. We report a case of Epirubicin extravasation seen at day 25 in a 46-year-old woman treated for breast cancer. A groin flap failed due to the persistence of anthracyclin in the wound. A split thickness skin graft was done after all the tendons were removed. The chemotherapy was interrupted for two months. Wide serial debridements are needed to achieve the removal of all molecules of anthracycline that are observed when granulating tissue is observed permanently in the wound.
\end{abstract}

Keywords: anthracycline; chemotherapy; debridement; extravasation; hand

Citation: Amouzou KS, Berny N, Haidara TM, Chlihi A, Ezzoubi M. Surgical management of a hand extravasation of anthracycline at late presentation. J Surg Dermatol 2019; 4(1): 111; http://dx.doi.org/10.18282/jsd.v2.i2.111.

*Correspondence to: Komla Sena Amouzou, Department of Surgery, University of Lomé, Lomé Togo; ksena.amouzou@gmail.com

Received: $21^{\text {st }}$ November 2018; Accepted: $8^{\text {th }}$ December 2018; Published Online: $20^{\text {th }}$ January 2019

\section{Introduction}

Anthracycline extravasation is a feared serious complication of intravenous chemotherapy. In absence of rapid treatment, progressive and extensive necrosis of skin and subcutaneous tissue, nerves, vessels, tendons, and joints can be seen ${ }^{[1,2]}$. Treatment of anthracycline extravasations is well codified nowadays. Surgical indications for anthracycline extravasation have, for the longest time, been liposuction, saline flush out, and early tissue excision. The discovery of the antidote (Dexrazoxane) has changed the lives of many patients. The use of Dexrazoxane has made invasive treatment rare ${ }^{[1-5]}$. Dexrazoxane is no longer suggested for patients with anthracycline extravasation at late presentation. Several debridements are then required. Removal of all molecules of anthracycline should be done prior to re- construction in order to achieve healing ${ }^{[1]}$.

In the hand and wrist, debridement after anthracycline extravasation is a great challenge. The dilemma is between hand function preservation and the total removal of anthracycline molecules. Necrosis of tissues can spread to the joints and bones if molecules of anthracycline remain on the site of extravasation ${ }^{[1,2,6]}$.

We report a case of hand anthracycline extravasation at late presentation and on the challenges of surgical management.

\section{Case report}

A 46-year-old female patient with type II diabetes for almost 15 years with oral treatment; and recently discovered hypertension under monotherapy, as medical history, was referred to our plastic and reconstructive department 25 days post a left-hand extravasation of Epirubicin. 
She presented with left breast stage III cancer treated by a Patey mastectomy and axillary node dissection. Adjuvant chemotherapy protocol was made of Epirubicin, cyclophosphamide, and fluorouracil. During the first stage, a flexible catheter $20 \mathrm{G}$ was used in the left anatomical snuff-box (the same side as axillary node dissection). This led to the extravasation of about $40 \mathrm{~mL}$ of Epirubicin. Early symptoms were pain, swelling, erythema. The catheter was removed, and cooling with a cold patch was done. Level 2 analgesics and topical corticosteroid were given to the patient. The hand was kept elevated above heart level. The hand was still painful and swelling continued. The appearance of induration and ulceration of skin motivated the consultation with our Plastic and Reconstructive Department 25 days post-accident.

Clinical findings were swelling, induration, and skin ulceration extending to the forearm (Figure 1). The range of motion of wrist and fingers were limited by pain. Tactile sensibility of the hand was subnormal. The patient had given consent for a surgical procedure.

Under the axillary block nerve, the infiltrated tissues of the dorsal hand and forearm were excised. The site remained detersive with inflammation of the surrounding $48 \mathrm{~h}$ post-surgical procedure. After serial debridements, we performed a groin pedicled flap (Figure 2). However, the procedure failed, and when the flap had been removed, the wound was still detersive. Finally, all the extensors were removed. A granulated tissue appeared. A split thickness skin graft was performed (Figure 3).

The patient's hospital stay in our department was two months. The patient kept a fixed wrist and fingers with a very low level of motion (Figure 3). Chemotherapy was stopped during the surgical management and was continued, after the skin graft was performed, by inserting a central venous access device. Early hand physiotherapy was not possible. The patient had a low pain threshold, was psychologically affected and was unable to tolerate physiotherapy.

\section{Discussion}

The vesicant properties of anthracyclines make them harmful to extravascular tissues. Since the approval of Dexrazoxane as an anthracycline antidote, surgical treatment has become rare ${ }^{[1-4]}$. The use of early treatment combination with saline flush out may be of good results, according to some authors ${ }^{[1]}$.

Late presentation management is still a matter of debate. It is known that all infiltrated tissues should be removed by surgical debridement ${ }^{[1,6]}$. Due to the fluores-

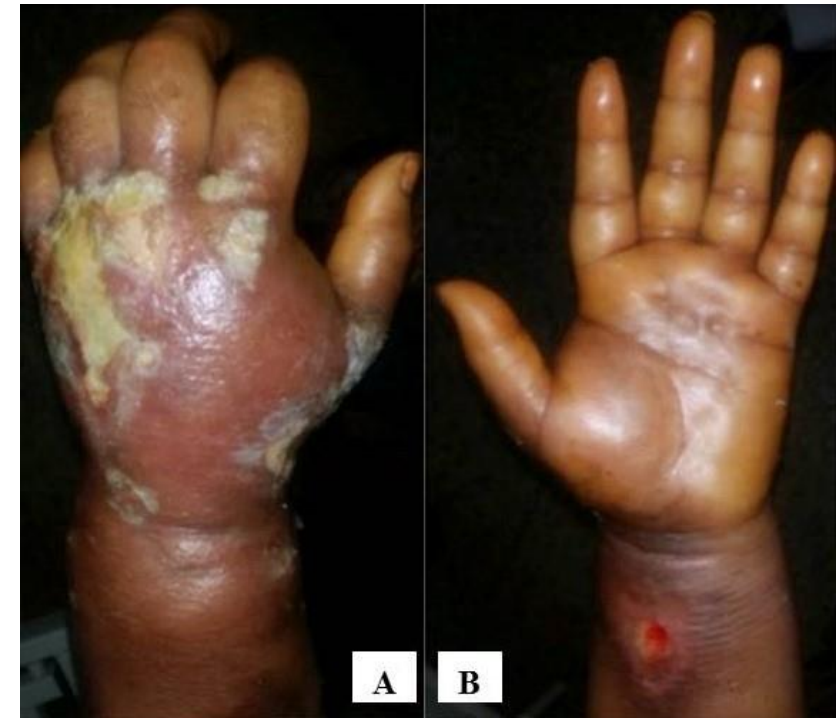

Figure 1. Clinical presentation of the Epirubicin extravasation at day 25: (A) Dorsal view, (B) Anterior view

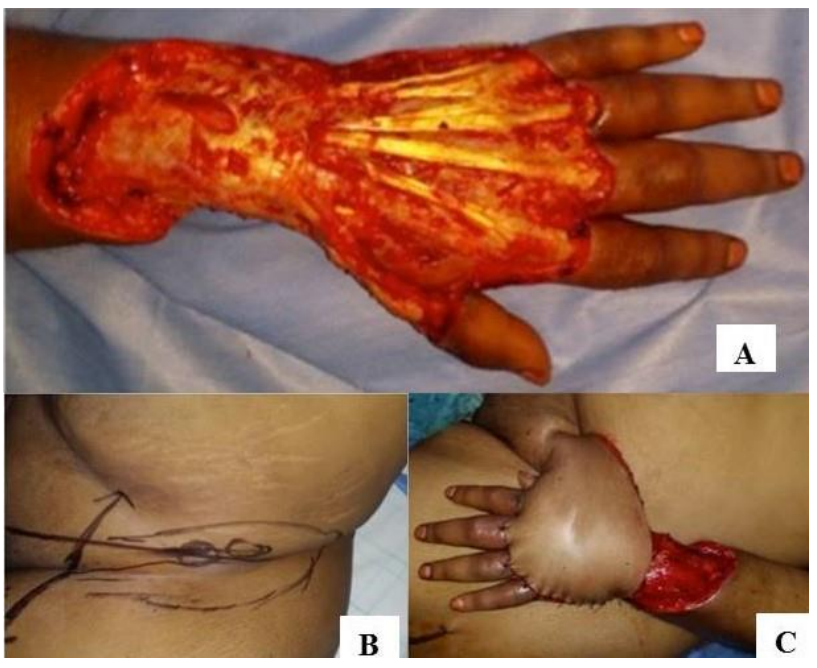

Figure 2. (A) Clinical presentation after six debridement; (B) groin flap design; (C) groin flap application to the hand

cent properties of anthracycline molecules, some surgeons perform debridement under a fluorescent microscope. However, anthracycline loses its fluorescent properties within 24 to 27 days $^{[1,7]}$. It is known that anthracyclines can stay in the site of extravasation for more than one year. Tissue damage will continue until the molecules of anthracycline totally disappear ${ }^{[1,2]}$. This is challenging at later stages because it is difficult to preoperatively detect the persistence of anthracyclines in tendons. When trying to preserve tendons, we kept anthracycline on the site of extravasation, which led to the failure of the flap. The persistence of anthracycline makes wounds appear detersive with fibrinous deposit, 


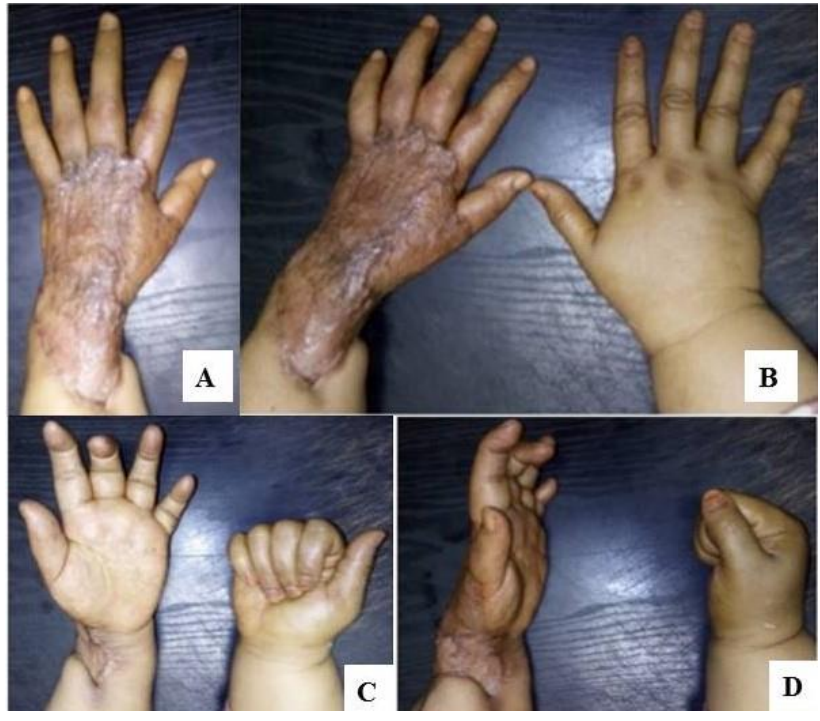

Figure 3. (A) Skin graft and wound healing; (B and C) Left hand and hand motion compared to the right hand; (D) Wrist ankylosis and hand motion limitation

without any granulating tissue formation ${ }^{[1]}$. Biopsy of the wound may be useful to confirm the presence of anthracycline but it requires toxicology tests that are not currently available in our setting. Poor results from hand reconstruction were linked with probable joints damage by anthracycline molecules and long managing time.

\section{Conclusion}

Severe tissue damage may be caused by anthracycline extravasation especially when diagnosed late. Wide serial debridements are needed to achieve removal of all molecules of anthracycline before any granulating tissue is observed permanently in the wound.

\section{Author contributions}

The corresponding author, KS Amouzou was responsible for the study design and concept, literature search, and writing, editing and review of the manuscript. N Berny was involved in data acquisition, literature search and manuscript review. TM Haidara contributed to manuscript editing and review while A Chlihi was involved in study concept and design. M Ezzoubi was involved in the study concept and design, definition of intellectual concept, as well as being the guarantor for this study.

\section{Conflict of interest}

The authors declare no potential conflict of interest with respect to the research, authorship, and/or publication of this article.

\section{References}

1. Langer SW, Sehested M, Jensen PB. Anthracycline extravasation: A comprehensive review of experimental and clinical treatments. Tumori 2009; 95(3): 273-282.

2. Langer SW. Dexrazoxane for the treatment of chemotherapy-related side effects. Cancer Manag Res 2014; 6: 357-363. doi:10.2147/CMAR.S47238.

3. Dinoi GL, Saibene G, Re B, Balzarini A, et. al. Cytotoxic extravasation: An issue disappearing or a problem without solution? Tumori 2016; 102(3): 290-293. doi:10.5301/tj.5000486.

4. Arroyo PA, Perez RU, Feijoo MAF, Hernandez MAC. Good clinical and cost outcomes using dexrazoxane to treat accidental epirubicin extravasation. J Can Res Ther 2010; 6(4): 573- 574. doi:10.4103/0973-1482.77081.

5. Kreidieh FY, Moukadem HA, El Saghir NS. Overview, prevention and management of chemotherapy extravasation. World J Clin Oncol 2016; 7(1): 87-97. doi:10.53 06/wjco.v7.i1.87.

6. Schrijvers DL. Extravasation: A dreaded complication of chemotherapy. Ann Oncol 2003; 14(3): 26-30. doi:10.1093/annonc/mdg744.

7. Dahlstrom KK, Chenoufi HL, Daugaard S. Fluorescence microscopic demonstration and demarcation of doxorubicin extravasation experimental and clinical studies. Cancer 2006; 65(8): 1722-1726. doi: 10.10 02/1097-0142(19900415)65:8<1722::AID-CNCR282065 0810>3.0.CO;2-E. 